\title{
Perturbation bounds for eigenvalues of diagonalizable matrices and singular values
}

\section{Duanmei Zhou ${ }^{1 *}$, Guoliang Chen², Guoxing $\mathrm{Wu}^{3}$ and Xiaoyan Chen ${ }^{4}$}

${ }^{*}$ Correspondence:

gzzdm2008@163.com

${ }^{1}$ College of Mathematics and

Computer Science, Gannan Normal

University, 341000 Ganzhou,

People's Republic of China

Full list of author information is

available at the end of the article

\begin{abstract}
Perturbation bounds for eigenvalues of diagonalizable matrices are derived. Perturbation bounds for singular values of arbitrary matrices are also given. We generalize some existing results.
\end{abstract}

MSC: 65F15; 15A18; 65F35; 15A42

Keywords: perturbation bound; eigenvalue; diagonalizable matrix; singular value

\section{Introduction}

Many problems in science and engineering lead to eigenvalue and singular value problems for matrices. Perturbation bounds of eigenvalues and singular values play an important role in matrix computations. Let $S_{n}$ be the set of all $n$ ! permutations of $\{1,2, \ldots, n\}$. If $x=$ $\left(x_{1}, x_{2}, \ldots, x_{n}\right)$ and $\pi \in S_{n}$, then the vector $x_{\pi}$ is defined as $\left(x_{\pi(1)}, x_{\pi(2)}, \ldots, x_{\pi(n)}\right)$. A square matrix is called doubly stochastic if its elements are real nonnegative numbers and if the sum of the elements in each row and in each column is equal to 1 . Let $\mathbb{C}^{n \times n}$ be the set of $n \times n$ complex matrices. Let $A=\left(a_{i j}\right) \in \mathbb{C}^{n \times n}$, we use the notation (see $[1,2]$ )

$$
\begin{aligned}
& \|A\|_{p}=\left(\sum_{i, j=1}^{n}\left|a_{i j}\right|^{p}\right)^{\frac{1}{p}} \text { for } p \geq 0, \\
& \|A\|_{q, p}=\left(\sum_{j=1}^{n}\left(\sum_{k=1}^{n}\left|a_{k j}\right|^{q}\right)^{\frac{p}{q}}\right)^{\frac{1}{p}} \text { for } p>0, q>0, \frac{1}{p}+\frac{1}{q}=1 .
\end{aligned}
$$

Let $T \in \mathbb{C}^{n \times n}$ and assume that

$$
\Lambda^{(k)}=\operatorname{diag}\left(\lambda_{1}^{(k)}, \lambda_{2}^{(k)}, \ldots, \lambda_{n}^{(k)}\right) \in \mathbb{C}^{n \times n}, \quad k=1, \ldots, 4
$$

are diagonal matrices. In [3], the following classical result is given:

$$
\left\|\Lambda^{(1)} T \Lambda^{(2)}-\Lambda^{(3)} T \Lambda^{(4)}\right\|_{2}^{2} \geq s_{n}^{2}(T) \sum_{i=1}^{n}\left|\lambda_{i}^{(1)} \lambda_{\pi(i)}^{(2)}-\lambda_{i}^{(3)} \lambda_{\pi(i)}^{(4)}\right|^{2}
$$

\section{Springer}


for some $\pi \in S_{n}$, where $s_{n}(T)$ is the smallest singular value of $T$. The inequality has many applications in bounding the (relative) perturbation for eigenvalues and singular values, such as [4-6] and the references therein. We generalize (1.3) in Section 2.

Let $\lambda(A)$ denote the spectrum of matrix $A$. In 1970, Ikramov [7] defined the 'Hölder distance $d_{p}(\lambda(A), \lambda(B))$ between the spectra' of the matrices $A$ and $B$, which have the eigenvalues $\lambda_{1}, \lambda_{2}, \ldots, \lambda_{n}$ and $\mu_{1}, \mu_{2}, \ldots, \mu_{n}$, respectively, by the equation:

$$
d_{p}(\lambda(A), \lambda(B))=\min _{\pi \in S_{n}}\left(\sum_{i=1}^{n}\left|\lambda_{i}-\mu_{\pi(i)}\right|^{p}\right)^{\frac{1}{p}} .
$$

If $A$ and $B$ are Hermitian matrices and $1 \leq p<2$, [7] obtained

$$
d_{p}(\lambda(A), \lambda(B)) \leq\|A-B\|_{p}
$$

which partially generalizes the Hoffman-Wielandt theorem [8]. However, for normal matrices (1.5) can no longer be valid. The purpose of this paper is to obtain several inequalities similar to (1.5) for diagonalizable matrices. We exhibit some upper bounds and lower bounds for $d_{p}(\lambda(A), \lambda(B))$ of diagonalizable matrices $A$ and $B$ in Section 2.

Majorization is one of the most powerful techniques for deriving inequalities. We use majorization to get some perturbation bounds for singular values. For simplicity of the notations, in most cases in this paper the vectors in $\mathbb{R}^{n}$ are regarded as row vectors, but when they are multiplied by matrices we regard them as column vectors. Given a real vector $x=\left(x_{1}, x_{2}, \ldots, x_{n}\right) \in \mathbb{R}^{n}$, we rearrange its components as $x_{[1]} \geq x_{[2]} \geq \cdots \geq x_{[n]}$.

Definition 1.1 ([9], p.14) For $x=\left(x_{1}, x_{2}, \ldots, x_{n}\right), y=\left(y_{1}, y_{2}, \ldots, y_{n}\right) \in \mathbb{R}^{n}$, if

$$
\sum_{i=1}^{k} x_{[i]} \leq \sum_{i=1}^{k} y_{[i]}, \quad k=1,2, \ldots, n,
$$

then we say that $x$ is weakly majorized by $y$ and denote $x \prec_{w} y$. If $x \prec_{w} y$ and $\sum_{i=1}^{n} x_{i}=$ $\sum_{i=1}^{n} y_{i}$, then we say that $x$ is majorized by $y$ and denote $x \prec y$.

Let $s_{1} \geq s_{2} \geq \cdots \geq s_{n}$ and $\delta_{1} \geq \delta_{2} \geq \cdots \geq \delta_{n}$ be the singular values of the complex matrices $A=\left(a_{i j}\right) \in \mathbb{C}^{n \times n}$ and $B=\left(b_{i j}\right) \in \mathbb{C}^{n \times n}$, respectively. In [10], p.215, and [11], p.199, the following classical result is given:

$$
\sum_{i=1}^{n}\left|s_{i}-\delta_{i}\right|^{2} \leq \sum_{i, j=1}^{n}\left|a_{i j}-b_{i j}\right|^{2}
$$

We generalize the inequality (1.6) in Section 3.

\section{Perturbation bounds for eigenvalues of diagonalizable matrices}

Let $A \circ B$ denote the Hadamard product of matrices $A$ and $B$. $\|A\|$ denotes the spectral norm of matrix $A$. $A^{T}$ denotes the transpose of matrix $A$. For two $n$-square real matrices $A$, $B$, we write $A \leq_{e} B$ to mean that $B-A$ is (entrywise) nonnegative. For $A=\left(a_{i j}\right) \in \mathbb{C}^{n \times n}$ and a real number $t>0$, we denote $A^{|\circ| t} \equiv\left(\left|a_{i j}\right|^{t}\right) \in \mathbb{C}^{n \times n}$. Let $s_{n}(A)$ and $s_{1}(A)$ be the smallest and 
the largest singular values of $A$, respectively. The following entrywise inequalities involve the smallest and the largest singular values.

Lemma 2.1 ([9], p.52) Let $A \in \mathbb{C}^{n \times n}$ and let $p, q$ be real numbers with $0<p \leq 2$ and $q \geq 2$. Then there exist two doubly stochastic matrices $B, C \in \mathbb{C}^{n \times n}$ such that

$$
s_{n}(A)^{p} B \leq_{e} A^{|\circ| p}
$$

and

$$
A^{|\circ| q} \leq_{e} S_{1}(A)^{q} C
$$

Theorem 2.2 Let $T \in \mathbb{C}^{n \times n}$ and let $p, q$ be real numbers with $0<p \leq 2$ and $q \geq 2$. Assume that $\Lambda^{(k)}=\operatorname{diag}\left(\lambda_{1}^{(k)}, \lambda_{2}^{(k)}, \ldots, \lambda_{n}^{(k)}\right) \in \mathbb{C}^{n \times n}, k=1, \ldots, 4$, are diagonal matrices. Then there are permutations $\pi$ and $v$ of $S_{n}$ such that

$$
\left\|\Lambda^{(1)} T \Lambda^{(2)}-\Lambda^{(3)} T \Lambda^{(4)}\right\|_{p}^{p} \geq s_{n}^{p}(T) \sum_{i=1}^{n}\left|\lambda_{i}^{(1)} \lambda_{\pi(i)}^{(2)}-\lambda_{i}^{(3)} \lambda_{\pi(i)}^{(4)}\right|^{p}
$$

and

$$
\left\|\Lambda^{(1)} T \Lambda^{(2)}-\Lambda^{(3)} T \Lambda^{(4)}\right\|_{q}^{q} \leq s_{1}^{q}(T) \sum_{i=1}^{n}\left|\lambda_{i}^{(1)} \lambda_{v(i)}^{(2)}-\lambda_{i}^{(3)} \lambda_{v(i)}^{(4)}\right|^{q}
$$

Proof Set $T=\left(t_{i j}\right) \in \mathbb{C}^{n \times n}$. Then

$$
\begin{aligned}
\left\|\Lambda^{(1)} T \Lambda^{(2)}-\Lambda^{(3)} T \Lambda^{(4)}\right\|_{p}^{p} & =\sum_{i, j=1}^{n}\left|t_{i j}\right|^{p}\left|\lambda_{i}^{(1)} \lambda_{j}^{(2)}-\lambda_{i}^{(3)} \lambda_{j}^{(4)}\right|^{p} \\
& =e^{T}\left(T^{|\circ| p} \circ M\right) e,
\end{aligned}
$$

where $M=\left(\left|\lambda_{i}^{(1)} \lambda_{j}^{(2)}-\lambda_{i}^{(3)} \lambda_{j}^{(4)}\right|^{p}\right) \in \mathbb{C}^{n \times n}, e=(1,1, \ldots, 1)^{T} \in \mathbb{C}^{n}$. Applying inequality (2.1), we have

$$
T^{|\circ| p} \geq s_{n}^{p}(T) B
$$

where $B=\left(b_{i j}\right)$ is a doubly stochastic matrix. Then

$$
\left\|\Lambda^{(1)} T \Lambda^{(2)}-\Lambda^{(3)} T \Lambda^{(4)}\right\|_{p}^{p} \geq e^{T}\left(s_{n}^{p}(T) B \circ M\right) e=s_{n}^{p}(T) e^{T}(B \circ M) e .
$$

Since $B$ is doubly stochastic, by Birkhoff's theorem ([12, 13], p.527) $B$ is a convex combination of permutation matrices:

$$
B=\sum_{i=1}^{n !} \tau_{i} P_{i}, \quad \tau_{i} \geq 0, \sum_{i=1}^{n !} \tau_{i}=1, P_{i} \text { are permutation matrices. }
$$


Suppose $e^{T}\left(B \circ P_{k}\right) e=\min \left\{e^{T}\left(B \circ P_{i}\right) e \mid 1 \leq i \leq n !\right\}$ and $P_{k}$ corresponds to $\pi \in S_{n}$. Then

$$
\begin{aligned}
\left\|\Lambda^{(1)} T \Lambda^{(2)}-\Lambda^{(3)} T \Lambda^{(4)}\right\|_{p}^{p} & \geq s_{n}^{p}(T) e^{T}(B \circ M) e \\
& =s_{n}^{p}(T) e^{T}\left(\sum_{i=1}^{n !} \tau_{i} P_{i} \circ M\right) e \\
& \geq s_{n}^{p}(T) \sum_{i=1}^{n !} \tau_{i} e^{T}\left(P_{k} \circ M\right) e \\
& =s_{n}^{p}(T) e^{T}\left(P_{k} \circ M\right) e \\
& =s_{n}^{p}(T) \sum_{i=1}^{n}\left|\lambda_{i}^{(1)} \lambda_{\pi(i)}^{(2)}-\lambda_{i}^{(3)} \lambda_{\pi(i)}^{(4)}\right|^{p} .
\end{aligned}
$$

Proving (2.3).

Use (2.2), (2.5), and the Birkhoff theorem, we can deduce the inequality (2.4).

Remark 2.3 If we take $p=2$, we get Theorem 3.2 in [3]. So, the bound in inequality (2.3) generalizes the bound of Theorem 3.2 in [3].

Next, we apply Theorem 2.2 to get some perturbation bounds for the eigenvalues of diagonalizable matrices. Let $A=\left(a_{i j}\right) \in \mathbb{C}^{n \times n}$ and $B=\left(b_{i j}\right) \in \mathbb{C}^{n \times n}$. When $p>0, q>0, \frac{1}{p}+$ $\frac{1}{q}=1$, then $($ see $[2])$

$$
\begin{aligned}
\|A B\|_{p} & \leq\|A\|_{p}\|B\|_{q, p}, \\
\|A B\|_{p} & \leq\left\|A^{T}\right\|_{q, p}\|B\|_{p} .
\end{aligned}
$$

If $B$ is nonsingular, then we have

$$
\frac{\|A\|_{p}}{\left\|B^{-1}\right\|_{q, p}} \leq\|A B\|_{p}
$$

If $A$ is nonsingular, then we have

$$
\frac{\|B\|_{p}}{\left\|\left(A^{-1}\right)^{T}\right\|_{q, p}} \leq\|A B\|_{p}
$$

For normal matrices the statement of Theorem 3 in [7] (inequality (1.5)) can no longer be valid. However, we have the following theorem.

Theorem 2.4 Assume that both $A \in \mathbb{C}^{n \times n}$ and $B \in \mathbb{C}^{n \times n}$ are diagonalizable and admit the following decompositions:

$$
A=D_{1} \Lambda_{1} D_{1}^{-1} \text { and } B=D_{2} \Lambda_{2} D_{2}^{-1}
$$


where $D_{1}$ and $D_{2}$ are nonsingular, and $\Lambda_{1}=\operatorname{diag}\left(\lambda_{1}, \lambda_{2}, \ldots, \lambda_{n}\right)$ and $\Lambda_{2}=\operatorname{diag}\left(\mu_{1}, \mu_{2}, \ldots\right.$, $\left.\mu_{n}\right)$. Then there are permutations $\pi$ and $v$ of $S_{n}$ such that

$$
\begin{aligned}
& \left(\sum_{i=1}^{n}\left|\lambda_{i}-\mu_{\pi(i)}\right|^{p}\right)^{\frac{1}{p}} \leq\left\|\left(D_{1}^{-1}\right)^{T}\right\|_{q, p}\left\|D_{2}\right\|_{q, p}\left\|D_{2}^{-1}\right\|\left\|D_{1}\right\|\|A-B\|_{p}, \\
& \left(\sum_{i=1}^{n}\left|\lambda_{i}-\mu_{v(i)}\right|^{p}\right)^{\frac{1}{p}} \leq\left\|\left(D_{2}^{-1}\right)^{T}\right\|_{q, p}\left\|D_{1}\right\|_{q, p}\left\|D_{1}^{-1}\right\|\left\|D_{2}\right\|\|A-B\|_{p},
\end{aligned}
$$

where $1<p \leq 2$ and $\frac{1}{p}+\frac{1}{q}=1$.

Proof Using (2.10), we have

$$
\begin{aligned}
\|A-B\|_{p}^{p} & =\left\|D_{1} \Lambda_{1} D_{1}^{-1}-D_{2} \Lambda_{2} D_{2}^{-1}\right\|_{p}^{p} \\
& =\left\|D_{1}\left(\Lambda_{1} D_{1}^{-1} D_{2}-D_{1}^{-1} D_{2} \Lambda_{2}\right) D_{2}^{-1}\right\|_{p}^{p}
\end{aligned}
$$

and

$$
\begin{aligned}
\|A-B\|_{p}^{p} & =\left\|D_{1} \Lambda_{1} D_{1}^{-1}-D_{2} \Lambda_{2} D_{2}^{-1}\right\|_{p}^{p} \\
& =\left\|D_{2}\left(D_{2}^{-1} D_{1} \Lambda_{1}-\Lambda_{2} D_{2}^{-1} D_{1}\right) D_{1}^{-1}\right\|_{p}^{p} .
\end{aligned}
$$

We give a proof of (2.11) with the help of (2.13). Similarly one can prove (2.12) using (2.14). Applying (2.8) and (2.9) to (2.13) we obtain

$$
\|A-B\|_{p}^{p} \geq \frac{\left\|\Lambda_{1} D_{1}^{-1} D_{2}-D_{1}^{-1} D_{2} \Lambda_{2}\right\|_{p}^{p}}{\left\|\left(D_{1}^{-1}\right)^{T}\right\|_{q, p}^{p}\left\|D_{2}\right\|_{q, p}^{p}}
$$

Using inequality (2.3), there is a permutation $\pi$ of $S_{n}$ such that

$$
\left\|\Lambda_{1} D_{1}^{-1} D_{2}-D_{1}^{-1} D_{2} \Lambda_{2}\right\|_{p}^{p} \geq s_{n}^{p}\left(D_{1}^{-1} D_{2}\right) \sum_{i=1}^{n}\left|\lambda_{i}-\mu_{\pi(i)}\right|^{p} .
$$

So we have

$$
\sum_{i=1}^{n}\left|\lambda_{i}-\mu_{\pi(i)}\right|^{p} \leq \frac{\left\|\left(D_{1}^{-1}\right)^{T}\right\|_{q, p}^{p}\left\|D_{2}\right\|_{q, p}^{p}}{s_{n}^{p}\left(D_{1}^{-1} D_{2}\right)}\|A-B\|_{p}^{p} .
$$

We use the relations

$$
s_{n}^{-1}\left(D_{1}^{-1} D_{2}\right)=\left\|\left(D_{1}^{-1} D_{2}\right)^{-1}\right\| \leq\left\|D_{1}\right\|\left\|D_{2}^{-1}\right\|
$$

to get the inequality in (2.11).

Theorem 2.5 Under the hypotheses of Theorem 2.4, there are permutations $\pi$ and $v$ of $S_{n}$ such that

$$
\left(\sum_{i=1}^{n}\left|\lambda_{i}-\mu_{\pi(i)}\right|^{q}\right)^{\frac{1}{q}} \geq \frac{\|A-B\|_{q}}{\left\|\left(D_{1}\right)^{T}\right\|_{p, q}\left\|D_{2}^{-1}\right\|_{p, q}\left\|D_{1}^{-1}\right\|\left\|D_{2}\right\|},
$$




$$
\left(\sum_{i=1}^{n}\left|\lambda_{i}-\mu_{\nu(i)}\right|^{q}\right)^{\frac{1}{q}} \geq \frac{\|A-B\|_{q}}{\left\|\left(D_{2}\right)^{T}\right\|_{p, q}\left\|D_{1}^{-1}\right\|_{p, q}\left\|D_{1}\right\|\left\|D_{2}^{-1}\right\|},
$$

where $q \geq 2$ and $\frac{1}{p}+\frac{1}{q}=1$.

Proof Using (2.10), we have

$$
\begin{aligned}
\|A-B\|_{q}^{q} & =\left\|D_{1} \Lambda_{1} D_{1}^{-1}-D_{2} \Lambda_{2} D_{2}^{-1}\right\|_{q}^{q} \\
& =\left\|D_{1}\left(\Lambda_{1} D_{1}^{-1} D_{2}-D_{1}^{-1} D_{2} \Lambda_{2}\right) D_{2}^{-1}\right\|_{q}^{q}
\end{aligned}
$$

and

$$
\begin{aligned}
\|A-B\|_{q}^{q} & =\left\|D_{1} \Lambda_{1} D_{1}^{-1}-D_{2} \Lambda_{2} D_{2}^{-1}\right\|_{q}^{q} \\
& =\left\|D_{2}\left(D_{2}^{-1} D_{1} \Lambda_{1}-\Lambda_{2} D_{2}^{-1} D_{1}\right) D_{1}^{-1}\right\|_{q}^{q} .
\end{aligned}
$$

Applying (2.6) and (2.7) to (2.17) we obtain

$$
\|A-B\|_{q}^{q} \leq\left\|\left(D_{1}\right)^{T}\right\|_{p, q}^{q}\left\|\Lambda_{1} D_{1}^{-1} D_{2}-D_{1}^{-1} D_{2} \Lambda_{2}\right\|_{q}^{q}\left\|D_{2}^{-1}\right\|_{p, q}^{q}
$$

Using inequality (2.4), there exists a permutation $\pi$ of $S_{n}$ such that

$$
\left\|\Lambda_{1} D_{1}^{-1} D_{2}-D_{1}^{-1} D_{2} \Lambda_{2}\right\|_{q}^{q} \leq s_{1}^{q}\left(D_{1}^{-1} D_{2}\right) \sum_{i=1}^{n}\left|\lambda_{i}-\mu_{\pi(i)}\right|^{q},
$$

so we have

$$
\sum_{i=1}^{n}\left|\lambda_{i}-\mu_{\pi(i)}\right|^{q} \geq \frac{\|A-B\|_{q}^{q}}{\left\|\left(D_{1}\right)^{T}\right\|_{p, q}^{q}\left\|D_{2}^{-1}\right\|_{p, q}^{q} s_{1}^{q}\left(D_{1}^{-1} D_{2}\right)} .
$$

We use the relations

$$
s_{1}\left(D_{1}^{-1} D_{2}\right)=\left\|D_{1}^{-1} D_{2}\right\| \leq\left\|D_{1}^{-1}\right\|\left\|D_{2}\right\|
$$

to get the inequality in (2.15).

The proof of inequality (2.16) is similar to the proof of (2.15) and is omitted here.

For $1 \leq p \leq 2$, it is well known [2] that the scalar function (1.1) of a matrix $A$ is a submultiplicative matrix norm. However, it is true for $0<p \leq 2$. Actually, according to the Cauchy-Schwarz inequality, we have

$$
\begin{aligned}
\|A B\|_{p}^{p} & =\sum_{i=1}^{n} \sum_{j=1}^{n}\left|\sum_{k=1}^{n} a_{i k} b_{k j}\right|^{p} \\
& \leq \sum_{i=1}^{n} \sum_{j=1}^{n}\left(\left(\sum_{k=1}^{n}\left|a_{i k}\right|^{2}\right)^{\frac{1}{2}}\left(\sum_{k=1}^{n}\left|b_{k j}\right|^{2}\right)^{\frac{1}{2}}\right)^{p} .
\end{aligned}
$$


Since for fixed vector $x=\left(x_{1}, x_{2}, \ldots, x_{n}\right)$, the function $p \rightarrow\left(\left|x_{1}\right|^{p}+\left|x_{2}\right|^{p}+\cdots+\left|x_{n}\right|^{p}\right)^{\frac{1}{p}}$ is decreasing on $(0, \infty)$,

$$
\begin{aligned}
\|A B\|_{p}^{p} & \leq \sum_{i=1}^{n} \sum_{j=1}^{n}\left(\left(\sum_{k=1}^{n}\left|a_{i k}\right|^{p}\right)\left(\sum_{k=1}^{n}\left|b_{k j}\right|^{p}\right)\right) \\
& =\left(\sum_{i=1}^{n} \sum_{k=1}^{n}\left|a_{i k}\right|^{p}\right)\left(\sum_{j=1}^{n} \sum_{k=1}^{n}\left|b_{k j}\right|^{p}\right) \\
& =\|A\|_{p}^{p}\|B\|_{p}^{p} .
\end{aligned}
$$

That is,

$$
\|A B\|_{p} \leq\|A\|_{p}\|B\|_{p}
$$

If $B$ is nonsingular, then

$$
\|A\|_{p}=\left\|A B B^{-1}\right\|_{p} \leq\|A B\|_{p}\left\|B^{-1}\right\|_{p} .
$$

So we have

$$
\frac{\|A\|_{p}}{\left\|B^{-1}\right\|_{p}} \leq\|A B\|_{p}
$$

Similarly, when $A$ is nonsingular, then

$$
\|B\|_{p}=\left\|A^{-1} A B\right\|_{p} \leq\left\|A^{-1}\right\|_{p}\|A B\|_{p} .
$$

That is,

$$
\frac{\|B\|_{p}}{\left\|A^{-1}\right\|_{p}} \leq\|A B\|_{p}
$$

Theorem 2.6 Under the assumptions of Theorem 2.4, there are permutations $\pi$ and $\nu$ of $S_{n}$ such that

$$
\begin{aligned}
& \left(\sum_{i=1}^{n}\left|\lambda_{i}-\mu_{\pi(i)}\right|^{p}\right)^{\frac{1}{p}} \leq\left\|D_{1}^{-1}\right\|_{p}\left\|D_{2}\right\|_{p}\left\|D_{2}^{-1}\right\|\left\|D_{1}\right\|\|A-B\|_{p}, \\
& \left(\sum_{i=1}^{n}\left|\lambda_{i}-\mu_{v(i)}\right|^{p}\right)^{\frac{1}{p}} \leq\left\|D_{2}^{-1}\right\|_{p}\left\|D_{1}\right\|_{p}\left\|D_{1}^{-1}\right\|\left\|D_{2}\right\|\|A-B\|_{p},
\end{aligned}
$$

where $0<p \leq 2$.

Proof The proof is similar to the proof of Theorem 2.4 and is omitted here.

Remark 2.7 Since

$$
\|A\|_{q, p}=\left(\sum_{j=1}^{n}\left(\sum_{k=1}^{n}\left|a_{k j}\right|^{q}\right)^{\frac{p}{q}}\right)^{\frac{1}{p}} \leq\left(\sum_{j=1}^{n}\left(\sum_{k=1}^{n}\left|a_{k j}\right|^{p}\right)^{\frac{p}{p}}\right)^{\frac{1}{p}}=\|A\|_{p}
$$


and

$$
\left\|A^{T}\right\|_{q, p} \leq\left\|A^{T}\right\|_{p}=\|A\|_{p}
$$

for $1<p \leq 2$ and $\frac{1}{p}+\frac{1}{q}=1$, the bounds in (2.11) and (2.12) are always sharper than those in (2.22) and (2.23), respectively.

When $p=q=2$, then $\|A B\|_{2} \leq \min \left\{\|A\|_{2}\|B\|,\|A\|\|B\|_{2}\right\}$. We obtain

$$
\begin{aligned}
\left(\sum_{i=1}^{n}\left|\lambda_{i}-\mu_{\pi(i)}\right|^{2}\right)^{\frac{1}{2}} & \leq\left\|D_{1}^{-1}\right\|\left\|D_{2}\right\|\left\|D_{2}^{-1} D_{1}\right\|\|A-B\|_{2} \\
& \leq\left\|D_{1}^{-1}\right\|\left\|D_{2}\right\|\left\|D_{2}^{-1}\right\|\left\|D_{1}\right\|\|A-B\|_{2}, \\
\left(\sum_{i=1}^{n}\left|\lambda_{i}-\mu_{\nu(i)}\right|^{2}\right)^{\frac{1}{2}} & \leq\left\|D_{2}^{-1}\right\|\left\|D_{1}\right\|\left\|D_{1}^{-1} D_{2}\right\|\|A-B\|_{2} \\
& \leq\left\|D_{2}^{-1}\right\|\left\|D_{1}\right\|\left\|D_{1}^{-1}\right\|\left\|D_{2}\right\|\|A-B\|_{2} .
\end{aligned}
$$

Since

$$
\|A\|_{p, q}=\left(\sum_{j=1}^{n}\left(\sum_{k=1}^{n}\left|a_{k j}\right|^{p}\right)^{\frac{q}{p}}\right)^{\frac{1}{q}} \leq\left(\sum_{j=1}^{n}\left(\sum_{k=1}^{n}\left|a_{k j}\right|^{p}\right)^{\frac{p}{p}}\right)^{\frac{1}{p}}=\|A\|_{p}
$$

and

$$
\left\|A^{T}\right\|_{p, q} \leq\left\|A^{T}\right\|_{p}=\|A\|_{p}
$$

for $q \geq 2$ and $\frac{1}{p}+\frac{1}{q}=1$, we have the following corollary.

Corollary 2.8 Under the same conditions as in Theorem 2.4, there are permutations $\pi$ and $v$ of $S_{n}$ such that

$$
\begin{aligned}
& \left(\sum_{i=1}^{n}\left|\lambda_{i}-\mu_{\pi(i)}\right|^{q}\right)^{\frac{1}{q}} \geq \frac{\|A-B\|_{q}}{\left\|D_{1}\right\|_{p}\left\|D_{2}^{-1}\right\|_{p}\left\|D_{1}^{-1}\right\|\left\|D_{2}\right\|} \\
& \left(\sum_{i=1}^{n}\left|\lambda_{i}-\mu_{\nu(i)}\right|^{q}\right)^{\frac{1}{q}} \geq \frac{\|A-B\|_{q}}{\left\|D_{1}^{-1}\right\|_{p}\left\|D_{2}\right\|_{p}\left\|D_{1}\right\|\left\|D_{2}^{-1}\right\|}
\end{aligned}
$$

where $q \geq 2$ and $\frac{1}{p}+\frac{1}{q}=1$.

Remark 2.9 When $p=q=2$, we obtain

$$
\begin{aligned}
& \left(\sum_{i=1}^{n}\left|\lambda_{i}-\mu_{\pi(i)}\right|^{2}\right)^{\frac{1}{2}} \geq \frac{\|A-B\|_{2}}{\left\|D_{1}\right\|\left\|D_{2}^{-1}\right\|\left\|D_{1}^{-1} D_{2}\right\|} \geq \frac{\|A-B\|_{2}}{\left\|D_{1}\right\|\left\|D_{2}^{-1}\right\|\left\|D_{1}^{-1}\right\|\left\|D_{2}\right\|}, \\
& \left(\sum_{i=1}^{n}\left|\lambda_{i}-\mu_{\nu(i)}\right|^{2}\right)^{\frac{1}{2}} \geq \frac{\|A-B\|_{2}}{\left\|D_{1}^{-1}\right\|\left\|D_{2}\right\|\left\|D_{2}^{-1} D_{1}\right\|} \geq \frac{\|A-B\|_{2}}{\left\|D_{1}^{-1}\right\|\left\|D_{2}\right\|\left\|D_{1}\right\|\left\|D_{2}^{-1}\right\|} .
\end{aligned}
$$




\section{Perturbation bounds for singular values}

For brevity we only consider square matrices. The generalizations from square matrices to rectangular matrices are obvious, and usually problems on singular values of rectangular matrices can be converted to the case of square matrices by adding zero rows or zero columns.

For a Hermitian matrix $G \in \mathbb{C}^{n \times n}$, we always denote $\lambda(G)=\left(\lambda_{1}(G), \lambda_{2}(G), \ldots, \lambda_{n}(G)\right)$, where $\lambda_{1}(G) \geq \lambda_{2}(G) \geq \cdots \geq \lambda_{n}(G)$ are the eigenvalues of $G$ in decreasing order.

Lemma 3.1 (Lidskii [14], Lemma 3.18 [9]) If $G, H \in \mathbb{C}^{n \times n}$ are Hermitian matrices, then

$$
\lambda(G)-\lambda(H) \prec \lambda(G-H) .
$$

Lemma 3.2 ([9], p.18) Let $f(t)$ be a convex function, $x=\left(x_{1}, x_{2}, \ldots, x_{n}\right), y=\left(y_{1}, y_{2}, \ldots, y_{n}\right) \in$ $\mathbb{R}^{n}$. Then

$$
x \prec y \quad \Longrightarrow \quad\left(f\left(x_{1}\right), f\left(x_{2}\right), \ldots, f\left(x_{n}\right)\right) \prec_{w}\left(f\left(y_{1}\right), f\left(y_{2}\right), \ldots, f\left(y_{n}\right)\right)
$$

Theorem 3.3 Let $\sigma_{1}(A) \geq \sigma_{2}(A) \geq \cdots \geq \sigma_{n}(A), \sigma_{1}(B) \geq \sigma_{2}(B) \geq \cdots \geq \sigma_{n}(B)$ and $\sigma_{1}(A-$ $B) \geq \sigma_{2}(A-B) \geq \cdots \geq \sigma_{n}(A-B)$ be the singular values of the complex matrices $A=\left(a_{i j}\right)$, $B=\left(b_{i j}\right)$ and $A-B$, respectively. Then

$$
\begin{aligned}
& \sum_{i=1}^{n}\left|\sigma_{i}(A)-\sigma_{i}(B)\right|^{p} \leq \sum_{i, j=1}^{n}\left|a_{i j}-b_{i j}\right|^{p}, \\
& \sum_{i=1}^{n}\left|\sigma_{i}(A)-\sigma_{i}(B)\right|^{q} \geq \sum_{i=1}^{n} \sigma_{i}^{q}(A-B),
\end{aligned}
$$

where $1 \leq p \leq 2,0<q \leq 1$.

Proof Let $\varphi(A) \triangleq\left(\begin{array}{cc}0 & A^{*} \\ A & 0\end{array}\right), \varphi(B) \triangleq\left(\begin{array}{cc}0 & B^{*} \\ B & 0\end{array}\right)$. Then

$$
\varphi(A-B)=\left(\begin{array}{cc}
0 & (A-B)^{*} \\
A-B & 0
\end{array}\right)
$$

$\varphi(A), \varphi(B), \varphi(A-B)$ are three Hermitian matrices. Assume that $U_{1}^{*} A V_{1}=\operatorname{diag}\left(\sigma_{1}(A), \sigma_{2}(A)\right.$, $\left.\ldots, \sigma_{n}(A)\right), U_{2}^{*} B V_{2}=\operatorname{diag}\left(\sigma_{1}(B), \sigma_{2}(B), \ldots, \sigma_{n}(B)\right)$ and $U_{3}^{*}(A-B) V_{3}=\operatorname{diag}\left(\sigma_{1}(A-B), \sigma_{2}(A-\right.$ $\left.B), \ldots, \sigma_{n}(A-B)\right)$ are singular value decompositions with $U_{1}, U_{2}, U_{3}, V_{1}, V_{2}, V_{3}$ unitary. Then

$$
Q_{1} \triangleq \frac{1}{\sqrt{2}}\left(\begin{array}{cc}
V_{1} & V_{1} \\
U_{1} & -U_{1}
\end{array}\right), \quad Q_{2} \triangleq \frac{1}{\sqrt{2}}\left(\begin{array}{cc}
V_{2} & V_{2} \\
U_{2} & -U_{2}
\end{array}\right) \quad \text { and } \quad Q_{3} \triangleq \frac{1}{\sqrt{2}}\left(\begin{array}{cc}
V_{3} & V_{3} \\
U_{3} & -U_{3}
\end{array}\right)
$$

are unitary matrices and

$$
\begin{aligned}
& Q_{1}^{*} \varphi(A) Q_{1}=\operatorname{diag}\left(\sigma_{1}(A), \sigma_{2}(A), \ldots, \sigma_{n}(A),-\sigma_{1}(A),-\sigma_{2}(A), \ldots,-\sigma_{n}(A)\right), \\
& Q_{2}^{*} \varphi(B) Q_{2}=\operatorname{diag}\left(\sigma_{1}(B), \sigma_{2}(B), \ldots, \sigma_{n}(B),-\sigma_{1}(B),-\sigma_{2}(B), \ldots,-\sigma_{n}(B)\right)
\end{aligned}
$$


and

$$
\begin{aligned}
Q_{3}^{*} \varphi(A-B) Q_{3}= & \operatorname{diag}\left(\sigma_{1}(A-B), \sigma_{2}(A-B), \ldots, \sigma_{n}(A-B),\right. \\
& \left.-\sigma_{1}(A-B),-\sigma_{2}(A-B), \ldots,-\sigma_{n}(A-B)\right) .
\end{aligned}
$$

By Lemma 3.1, we have

$$
\begin{aligned}
&\left(\sigma_{1}(A)-\sigma_{1}(B), \sigma_{2}(A)-\sigma_{2}(B), \ldots, \sigma_{n}(A)-\sigma_{n}(B),\right. \\
&\left.\quad \sigma_{n}(B)-\sigma_{n}(A), \ldots, \sigma_{2}(B)-\sigma_{2}(A), \sigma_{1}(B)-\sigma_{1}(A)\right) \\
& \prec\left(\sigma_{1}(A-B), \sigma_{2}(A-B), \ldots, \sigma_{n}(A-B),\right. \\
&\left.-\sigma_{n}(A-B), \ldots,-\sigma_{2}(A-B),-\sigma_{1}(A-B)\right) .
\end{aligned}
$$

First consider the case $1 \leq p \leq 2$. Since the function $f(t)=|t|^{p}$ is convex on $(-\infty,+\infty)$, applying Lemma 3.2 with $f(t)$ to the majorization (3.3) yields

$$
\begin{aligned}
& \left(\left|\sigma_{1}(A)-\sigma_{1}(B)\right|^{p},\left|\sigma_{2}(A)-\sigma_{2}(B)\right|^{p}, \ldots,\left|\sigma_{n}(A)-\sigma_{n}(B)\right|^{p},\right. \\
& \left.\quad\left|\sigma_{n}(B)-\sigma_{n}(A)\right|^{p}, \ldots,\left|\sigma_{2}(B)-\sigma_{2}(A)\right|^{p},\left|\sigma_{1}(B)-\sigma_{1}(A)\right|^{p}\right) \\
& \quad{ }_{w}\left(\sigma_{1}^{p}(A-B), \sigma_{2}^{p}(A-B), \ldots, \sigma_{n}^{p}(A-B), \sigma_{n}^{p}(A-B), \ldots, \sigma_{2}^{p}(A-B), \sigma_{1}^{p}(A-B)\right) .
\end{aligned}
$$

In particular,

$$
\begin{aligned}
& \left(\left|\sigma_{1}(A)-\sigma_{1}(B)\right|^{p},\left|\sigma_{2}(A)-\sigma_{2}(B)\right|^{p}, \ldots,\left|\sigma_{n}(A)-\sigma_{n}(B)\right|^{p}\right) \\
& \quad{ }_{w}\left(\sigma_{1}^{p}(A-B), \sigma_{2}^{p}(A-B), \ldots, \sigma_{n}^{p}(A-B)\right) .
\end{aligned}
$$

According to Theorem 1 of [15] or Theorem 3.32 of [9], we have

$$
\sum_{i=1}^{n} \sigma_{i}^{p}(A-B) \leq \sum_{i, j=1}^{n}\left|a_{i j}-b_{i j}\right|^{p}
$$

for $1 \leq p \leq 2$. Combining (3.4) and (3.5), we obtain (3.1).

When $0<q \leq 1$, by considering the convex function $g(t)=-|t|^{q}$, on $(-\infty,+\infty)$, applying Lemma 3.2 with $g(t)$ to the majorization (3.3) yields

$$
\begin{aligned}
& \left(-\left|\sigma_{1}(A)-\sigma_{1}(B)\right|^{q}, \ldots,-\left|\sigma_{n}(A)-\sigma_{n}(B)\right|^{q},-\left|\sigma_{n}(B)-\sigma_{n}(A)\right|^{q}, \ldots,-\left|\sigma_{1}(B)-\sigma_{1}(A)\right|^{q}\right) \\
& \quad{ }_{w}\left(-\sigma_{1}^{q}(A-B), \ldots,-\sigma_{n}^{q}(A-B),-\sigma_{n}^{q}(A-B), \ldots,-\sigma_{1}^{q}(A-B)\right) .
\end{aligned}
$$

In particular,

$$
\begin{aligned}
& \left(-\left|\sigma_{1}(A)-\sigma_{1}(B)\right|^{q},-\left|\sigma_{2}(A)-\sigma_{2}(B)\right|^{q}, \ldots,-\left|\sigma_{n}(A)-\sigma_{n}(B)\right|^{q}\right) \\
& \quad{ }_{w}\left(-\sigma_{1}^{q}(A-B),-\sigma_{2}^{q}(A-B), \ldots,-\sigma_{n}^{q}(A-B)\right) .
\end{aligned}
$$

From (3.6), we get (3.2).

Remark 3.4 From inequality (3.1), if $p=2$, we get the inequality (1.6). So the inequality (3.1) generalizes the inequality (3.5.33) of [10], p.215, and Theorem 3.12 of [11], p.199. 
Competing interests

The authors declare that they have no competing interests.

\section{Authors' contributions}

All authors conceived of the study, participated in its design and coordination, drafted the manuscript, participated in the sequence alignment, and read and approved the final manuscript.

\section{Author details}

${ }^{1}$ College of Mathematics and Computer Science, Gannan Normal University, 341000 Ganzhou, People's Republic of China. ${ }^{2}$ Department of Mathematics, East China Normal University, 200241 Shanghai, People's Republic of China. ${ }^{3}$ Department of Mathematics, Northeast Forestry University, 150040 Harbin, People's Republic of China. ${ }^{4}$ Library, Gannan Normal University, 341000 Ganzhou, People's Republic of China.

\section{Acknowledgements}

This work is supported by the National Natural Science Foundation of China (No. 11501126, No. 11471122), the Youth Natural Science Foundation of Jiangxi Province (No. 20151BAB211011), the Science Foundation of Jiangxi Provincial Department of Education (No. GJJ150979), and the Supporting the Development for Local Colleges and Universities Foundation of China - Applied Mathematics Innovative team building.

Received: 19 January 2016 Accepted: 28 March 2016 Published online: 08 April 2016

\section{References}

1. Li, R: Norms of certain matrices with applications to variations of the spectra of matrices and matrix pencils. Linear Algebra Appl. 182, 199-234 (1993)

2. Ostrowski, A: Über Normen von Matrizen. Math. Z. 63, 2-18 (1955)

3. Elsner, L, Friedland, S: Singular value, doubly stochastic matrices, and applications. Linear Algebra Appl. 220, 161-169 (1995)

4. Chen, X: On perturbation bounds of generalized eigenvalues for diagonalizable pairs. Numer. Math. 107, 79-86 (2007)

5. Li, R: Spectral variations and Hadamard products: some problems. Linear Algebra Appl. 278, 317-326 (1998)

6. Li, W, Sun, W: Combined perturbation bounds: I. Eigensystems and singular value decompositions. SIAM J. Matrix Anal. Appl. 29, 643-655 (2007)

7. Ikramov, KhD: Some estimates for the eigenvalues of matrices. Zh. Vychisl. Mat. Mat. Fiz. 10(1), 172-177 (1970)

8. Hoffman, AJ, Wielandt, HW: The variation of the spectrum of a normal matrix. Duke Math. J. 20, 37-39 (1953)

9. Zhan, X: Matrix Inequalities. LNM, vol. 1790. Springer, Berlin (2002)

10. Horn, RA, Johnson, CR: Topics in Matrix Analysis. Cambridge University Press, Cambridge (1991)

11. Sun, JG: Matrix Perturbation Analysis. Science Press, Beijing (2001)

12. Birkhoff, G: Tres observaciones sobre el Algebra lineal. Rev. Univ. Nac. Tucumán Ser. A, Mat. Fis. Teor. 5, 147-151 (1946)

13. Horn, RA, Johnson, CR: Matrix Analysis. Cambridge University Press, Cambridge (1985)

14. Lidskii, VB: On the characteristic numbers of a sum and product of symmetric matrices. Dokl. Akad. Nauk SSSR 75, 769-772 (1950)

15. Ikramov, KhD: A simple proof of the generalized Schur inequality. Linear Algebra Appl. 199, 143-149 (1994)

\section{Submit your manuscript to a SpringerOpen ${ }^{\circ}$ journal and benefit from:}

- Convenient online submission

Rigorous peer review

- Immediate publication on acceptance

- Open access: articles freely available online

- High visibility within the field

- Retaining the copyright to your article 